\section{Herzinsuffizienz-Leitlinie entschlossen umsetzen}

- Vor einem Jahr wurde der $\mathrm{I}_{\mathrm{f}}$-Kanal-Hemmer Ivabradin als weitere Standardtherapie in die aktuelle ESC-Leitlinie zur chronischen Herzinsuffizienz aufgenommen. Jetzt sollte diese Behandlungsoption noch stärker im Praxisalltag implementiert werden.

Als wichtigste Behandlungsziele bei chronischer systolischer Herzinsuffizienz werden seitens der ESC die Besserung der Symptomatik sowie die Reduktion von Hospitalisierungen und der Mortalität postuliert. Als zusätzliche Eckpunkte werden die Erhöhung der Lebensqualität und die Steigerung der Funktionskapazität hervorgehoben, erläuterte Prof. Pjotr Ponikowski vom Centre for Heart Diseases in Wroclaw (Polen). Um alle diese Vorgaben zu verwirklichen, bedarf es nach seinen Worten einer stufenwei- sen Therapieeskalation unter Nutzung aller empfohlenen Medikamente. Ist die neurohumorale Schiene mit ACE-Hemmer, Betablocker und Mineralokortikoidrezeptor-Antagonisten ausgereizt, sollten entsprechend der ESC-Leitlinie symptomatische Patienten (NYHA-Klasse II-IV) im Sinusrhythmus, einer linksventrikulären Auswurffraktion $\leq 35 \%$ und einer Herzfrequenz $\geq 70$ /Minute konsequent und vor allem rasch auf Ivabradin (Procoralan ${ }^{\circledast}$ ) eingestellt werden. In der Praxis ist laut Ponikowski mit Ivabradin rasch eine Herzfrequenz von 60/Minute anzustreben, deren Erreichen in der $\mathrm{SHI}_{\mathrm{f}} \mathrm{T}$-Studie den größten therapeutischen Vorteil zur Folge hatte.

Die Evidenz für Ivabradin ist absolut überzeugend, betonte Prof. Michel Komajda vom Institute of Cardiology in Paris (Frankreich). Neben der signifikanten Reduktion der kardiovaskulären Mortalität legte der Kardiologe besonderes Augenmerk auf das verringerte Risiko wiederholter, kostenintensiver herzinsuffizienzassoziierter Krankenhausaufenthalte.

Nicht zuletzt ermöglicht Ivabradin auch eine deutliche Besserung der gesundheitsspezifischen Lebensqualität. Aktuelle Daten untermauern die Effektivität und Sicherheit von Ivabradin jetzt zusätzlich bei Patienten mit sehr schwerer Herzinsuffizienz (NYHAKlasse IV oder linksventrikulären Auswurffraktion < 20\%) und höherem Alter, so Komajda.

Dr. Michael Lohmann

Satellitensymposium, Heart Failure-Kongress, Lissabon (Portugal), 26.5.2013 (Veranstalter: Servier)

\section{TAVI gute Alternative zur Operation}

_ Der Anteil der Patienten über 80 Jahren, die einen Aortenklappenersatz benötigen, steigt seit Jahren. Fast ein Drittel dieser Patienten ist inoperabel. Für sie bietet sich eine interventionelle Prozedur am schlagenden Herzen an. Bei diesen Hochrisikopatienten hat sich eine Transkatheter-Aortenklappenimplantation (TAVI) als gleichwertige Alternative zur Operation erwiesen.

In der PARTNER-B-Studie konnte das katheterbasierte Verfahren die Mortalität der $\mathrm{Pa}$ tienten im Vergleich zur medikamentösen Standardtherapie signifikant senken. Auch die Lebensqualität verbesserte sich in der TAVI-Gruppe signifikant.

In der PARTNER-A-Studie zeigte sich hinsichtlich der Gesamtmortalität nach einem Jahr (24,3 vs. $26,8 \%$ ) kein Unterschied zwischen TAVI und Operation. Auch nach zwei und drei Jahren waren beide Gruppen vergleichbar.

Dr. Angelika Bischoff

Pressekonferenz, 79. Jahrestagung der Deutschen Gesellschaft für Kardiologie, Mannheim, 4.4.2013 (Veranstalter: Edwards Lifesciences)

\title{
Multi-Elektroden-Ablationssystem für die renale Denervierung
}

- Das Unternehmen St. Jude Medical hat für die endovaskulären Denervierung der sympathischen Nervenfasern in den Wänden der Nierenarterien das Multi-Elektroden-Ablations-System EnligHTN ${ }^{\mathrm{TM}}$ entwickelt, das die Eingriffszeit aufgrund der simultanen Ablation mehrerer Punkte deutlich zu reduzieren vermag. Beim EuroPCRKongress wurden erstmals 1-Jahres-Ergebnisse einer Pilotstudie mit 46 Patienten mit therapierefraktärer Hypertonie vorgestellt. Der Ausgangsblutdruck lag bei 176/96 $\mathrm{mmHg}$.
Ein Jahr nach der Behandlung war der Blutdruck im Mittel um 27/11 mmHg gesunken. $80 \%$ der Patienten sprachen mit einer Blutdrucksenkung von $>10 \mathrm{mmHg}$ an. Änderungen der Nierenfunktion wurden nicht beobachtet, berichtete Prof. Stephen Worthley, Royal Adelaide Hospital, Australien. Das Multi-Elektroden-Ablationssystem wird nun in einem großen Studienprogramm erforscht.

Dr. Dirk Einecke

Symposium, EuroPCR 2013, Paris, 23.5.2013 (Veranstalter: St. Jude Medical)

\section{Bessere INR-Werte bei Selbstmessung}

_ Laut Prof. Federico Tatò, München, haben Vitamin-K-Antagonisten nach wie vor einen hohen Stellenwert wenn eine orale Antikoagulation angezeigt ist. Engmaschiges Monitoring durch Gerinnungsselbstmanagement unterstützt die Optimierung idealer INR-Werte bei einer Therapie mit Vitamin-K-Antagonisten. Darüber hinaus bleibt die Therapie mit Vitamin-K-Antagonisten in Verbindung mit Gerinnungsselbstmanagement (z.B. CoaguCheck ${ }^{\circledast}$ ) eine kosteneffiziente Option zur Antikoagulation.

Dr. Ute Scholz, Zentrum für Blutgerinnungsstörungen, Leipzig, betonte, dass die INRWerte von Patienten, die diese eigenverantwortlich in regelmäßigen Abständen selbst gemessen hatten, sich deutlich häufiger (80\%) im therapeutischen Bereich befanden als die Werte von konventionell betreuten Patienten (65\%). Studienergebnissen zufolge können selbst schwerwiegende Komplikationen durch das Selbstmanagement signifikant reduziert werden. Das Gerinnungsselbstmanagement wurde 2010 in die Leitlinien der European Society of Cardiology (ESC) aufgenommen.

Dr. Yvette Zwick

Symposium beim 119. Kongress der Deutschen Gesellschaft für InnereMedizin (DGIM), Wiesbaden, 6.4.2013 (Veranstalter: Roche Diagnostics) 